\title{
Application of Data Smoothing Method in Signal Processing for Vortex Flow Meters
}

\author{
Jun ZHANG ${ }^{1}$, Tian ZOU ${ }^{2}$ and Chun-Lai TIAN ${ }^{1, a}$ \\ ${ }^{1}$ School of Mechanical and Electronic Engineeing, Pingxiang University, 337055 Pingxiang, Jiangxi Province, China \\ ${ }^{2}$ School of Law, Pingxiang University, 337055 Pingxiang, Jiangxi Province, China
}

\begin{abstract}
Vortex flow meter is typical flow measure equipment. Its measurement output signals can easily be impaired by environmental conditions. In order to obtain an improved estimate of the time-averaged velocity from the vortex flow meter, a signal filter method is applied in this paper. The method is based on a simple Savitzky-Golay smoothing filter algorithm. According with the algorithm, a numerical program is developed in Python with the scientific library numerical Numpy. Two sample data sets are processed through the program. The results demonstrate that the processed data is available accepted compared with the original data. The improved data of the time-averaged velocity is obtained within smoothing curves. Finally the simple data smoothing program is useable and stable for this filter.
\end{abstract}

\section{Introduction}

As types of flow measurement equipments, vortex flow meters are commonly used to measure the flow velocity. According with the advanced development of the flow measurement technology, they play a major role in flow measurement business. The vortex flow meters are extensively used in industries and academic research. It is being currently applied extensively in the measurement of liquids, gases and steam [1].

The vortex flow meter is a device that works with based on the principle of shedding of vortices behind a bluff body placed in the flow. Disturbances in the flow called vortices are created while the fluid passes this bluff body. The vortices trail behind the cylinder, appearing alternatively from each side of the body. The frequency at which these vortices alternate sides is proportional to the fluid flow rate. The flow velocity can be obtained from this frequency. The accuracy and reliability of the meters keeps them ahead of other devices [2-5].

Vortex flow meters are commonly employed in a variety of industry applications. However their robustness and accuracy can easily be impaired by environmental conditions, such as inflow disturbances and pulsating conditions. Many works on the signal post-processing have been carried out [4-8]. Some of them were based on mathematical filter methods to establish the data filter. And others built the filter software in the embedded computer of the vortex flow meters.

In order to obtain an improved estimate of the velocity from the meter, we apply a classical simple smoothing filter for the signal data post-processing in this paper. It is based the Savitzky-Golay filter and is corrected to adjust the fluctuation of the gas and reduce the lag of the transition.

\footnotetext{
${ }^{a}$ Corresponding author: tianchunlai@bit.edu.cn
} 


\section{Methodologies}

The Savitzky-Golay filter is a classical smoothing method for the signal processing [9]. It can be applied to a set of digital data points for the purpose of smoothing the data. And it helps to increase the signal-to-noise ratio without greatly distorting the signal. This is achieved, in a process known as convolution, by fitting successive sub-sets of adjacent data points with a low-degree polynomial by the method of linear least squares. The mathematic problem is described as the followings.

Assumed that there are the data consists of a set of $n\left\{x_{j}, y_{j}\right\}$ points $(\mathrm{j}=1, \ldots, n)$, where $x$ is an independent variable and $y_{j}$ is an observed value. They are treated with a set of $m$ convolution coefficients, $C_{i}$ according to the expression

$$
Y_{j}=\sum_{i=-(m-1) / 2}^{i=(m-1) / 2} C_{i} y_{j+i}
$$

The index $j$ represents the running index of the ordinate data in the original data table. It is easy to apply this formula in numerical program. Selected convolution coefficients could be found in the Savitzky-Golay tables [9].

For example, for smoothing by a 5-point quadratic polynomial, $m=5, i=\{-2,-1,0,1,2\}$ and $Y_{j}$ is given by

$$
Y_{j}=\frac{1}{35}\left(-3 \times y_{j-2}+12 \times y_{j-1}+17 \times y_{j}+12 \times y_{j+1}-3 \times y_{j+2}\right)
$$

where $C_{-2}=-3 / 35, C_{-1}=12 / 35, C_{0}=17 / 35, C_{1}=12 / 35, C_{2}=-3 / 35$.

Moreover such as another example, for smoothing by a 7-point quadratic polynomial, $m=7, i=\{-3$, $-2,-1,0,1,2,3\}$ and $Y_{j}$ is given by

$$
Y_{j}=\frac{1}{21}\left(-2 \times y_{j-3}+3 \times y_{j-2}+6 \times y_{j-1}+7 \times y_{j}+6 \times y_{j+1}+3 \times y_{j+2}-2 \times y_{j+3}\right)
$$

where $C_{-3}=-2 / 21, C_{-2}=3 / 21, C_{-1}=6 / 21, C_{0}=7 / 21, C_{1}=6 / 21, C_{2}=3 / 21, C_{3}=-2 / 21$.

\section{Solutions}

There is a simple application of data smoothing, which are performed primarily to make the data appear to be less noisy than it really is. According with above mathematic equations, the numerical solver is programmed within Numpy, a python scientific numerical library [10].

\section{Results and discussion}

Utilizing the solver, we obtain the processed data of two samples in normalized data. The original data of sample I are shown in Figure. 1. Its smoothed data processed by the filter with 5-point quadratic polynomial are shown in Figure. 2. And its smoothed data processed by the filter with 7-point quadratic polynomial are shown in Figure. 3. The other sample data set is shown in Figure. 4 (Original), Figure. 5 (filtered with 5-point quadratic polynomial) and Figure. 6 (filtered with 7-point quadratic polynomial).

Comparing these figures, it can be seen from Figure. 1 that there are some noise signals in the original data. In the Figure. 2, the processed results are smoothed through the filter of the noise with 5-point quadratic polynomial. And in the Figure. 3, the data are demonstrated with fewer noise data. It looks like as same as that in Figure. 4 to Figure. 6 with simple data II. It shows that the proposed smoothing algorithm is feasible for the vortex flow meters in the fluctuation conditions. Moreover the lag of the transition is reduced here. 


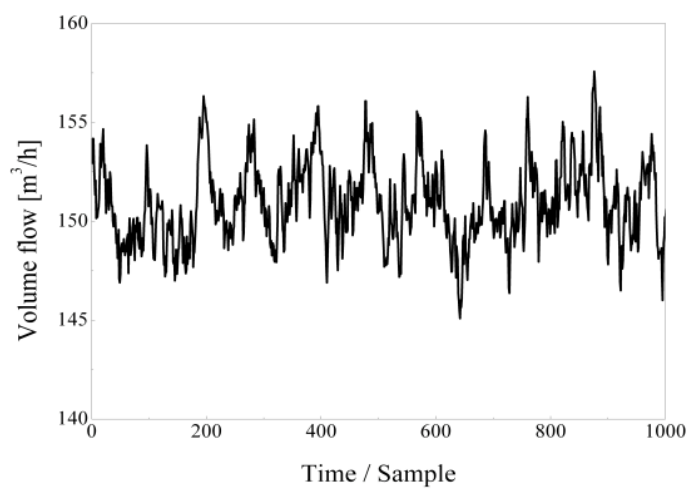

Figure 1. Original data I.

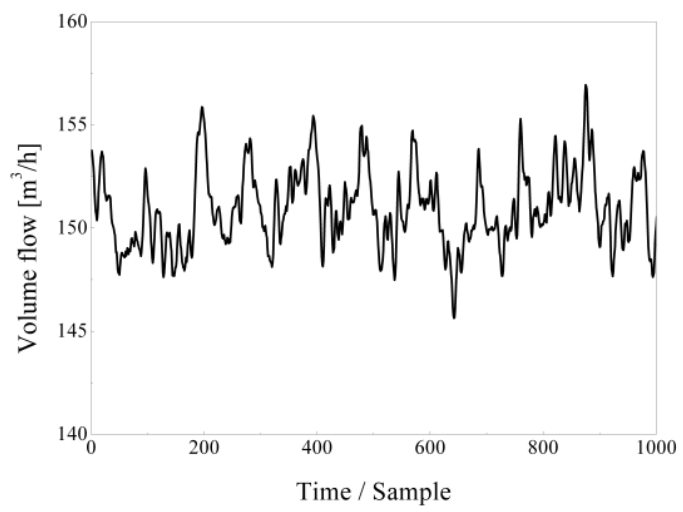

Figure 2. Processed data I by 5-point quadratic polynomial.

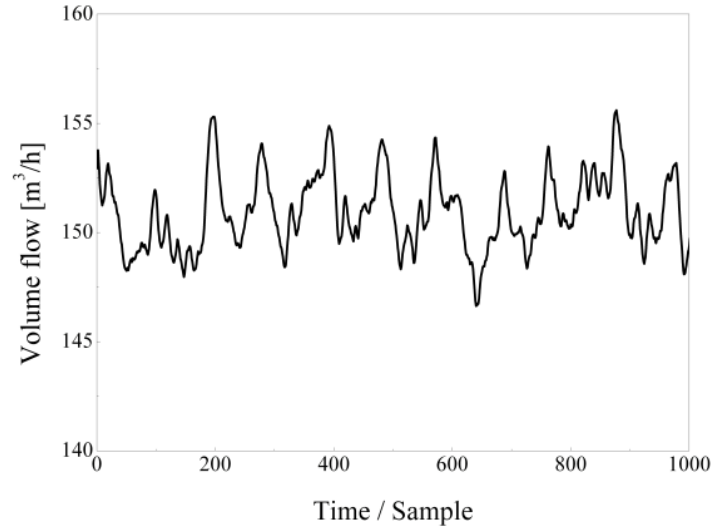

Figure 3. Processed data I by 7-point quadratic polynomial. 


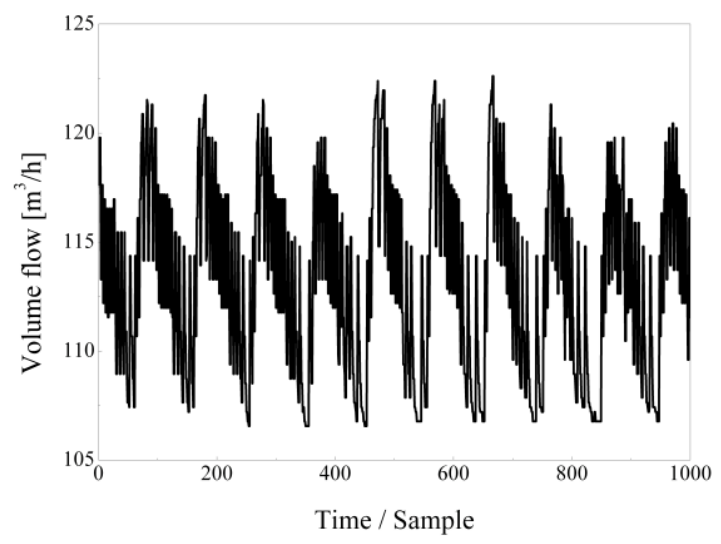

Figure 4. Original data II.

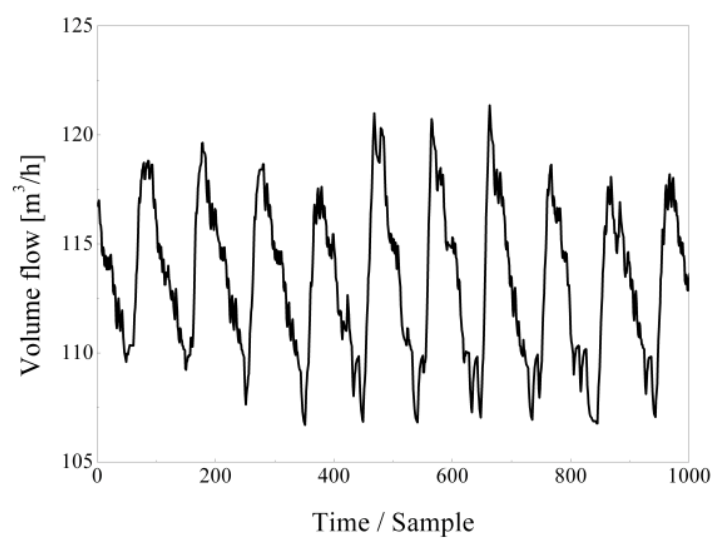

Figure 5. Processed data II by 5-point quadratic polynomial.

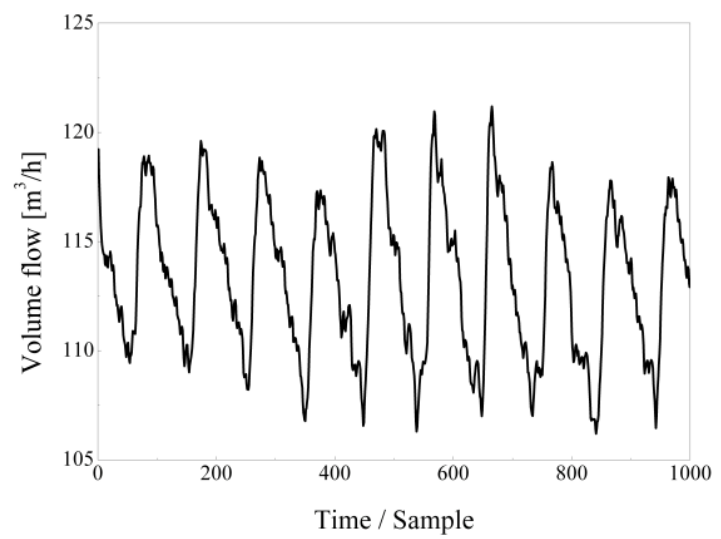

Figure 6. Processed data II by 7-point quadratic polynomial. 
The data results, total average and standard deviation with maximal normalization, are listed in Table 1. The processed data has the same average as the original data. In terms of the data filter for Data I, its average of Processed by 5-point quadratic polynomial is a little more than the original results and a little less than the results processed by 7-point quadratic polynomial filter. The standard deviation of the results processed by 7-point quadratic polynomial filter is the smallest due to the filter mathematical method.

Table 1. Average and deviation of two sample data

\begin{tabular}{|c|c|c|c|}
\hline \multirow{2}{*}{ Item } & Total average & Standard deviation \\
\hline \multirow{4}{*}{ Data I } & Original & 1.01060 & 0.01621 \\
\cline { 2 - 4 } & $\begin{array}{l}\text { Processed by 5-point } \\
\text { quadratic polynomial }\end{array}$ & 1.01020 & 0.01406 \\
\cline { 2 - 4 } & $\begin{array}{l}\text { Processed by 7-point } \\
\text { quadratic polynomial }\end{array}$ & 1.01030 & 0.01206 \\
\hline \multirow{5}{*}{ Data II } & $\begin{array}{l}\text { Original } \\
\text { Processed by 5-point } \\
\text { quadratic polynomial }\end{array}$ & 0.98114 & 0.03712 \\
\cline { 2 - 4 } & $\begin{array}{l}\text { Processed by 7-point } \\
\text { quadratic polynomial }\end{array}$ & 0.98104 & 0.02966 \\
\hline
\end{tabular}

Another data example with linear increase trend is shown in Figure. 7 with filtered results, which is shown in Figure. 8. In the Figure. 7 and Figure. 8, the dash lines display the linear increase trend. In the measurement, the trend of the gas or liquid flow would affect the meter data transition due to the system lag. The gradient of the linear fit for original data in Figure. 7 is 0.1505 . And that gradient of the filter processed data in Figure. 8 is 0.1504 , almost similarly with the original one. The little error is caused by some sample data changed by the filter.

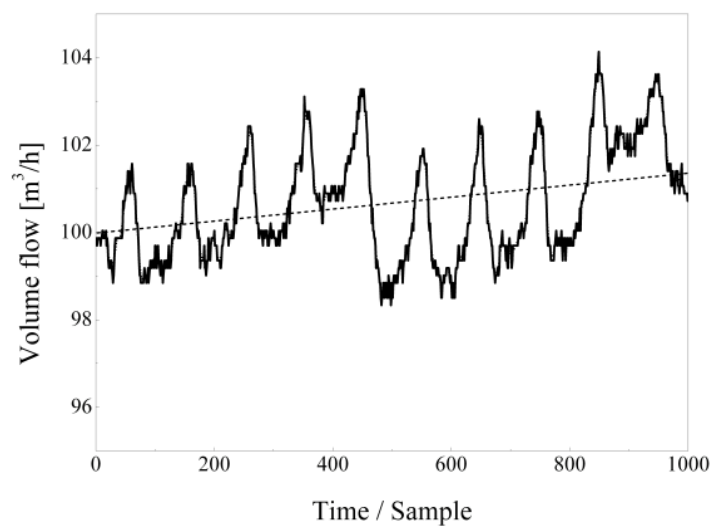

Figure 7. Original data III with linear increase trend. 


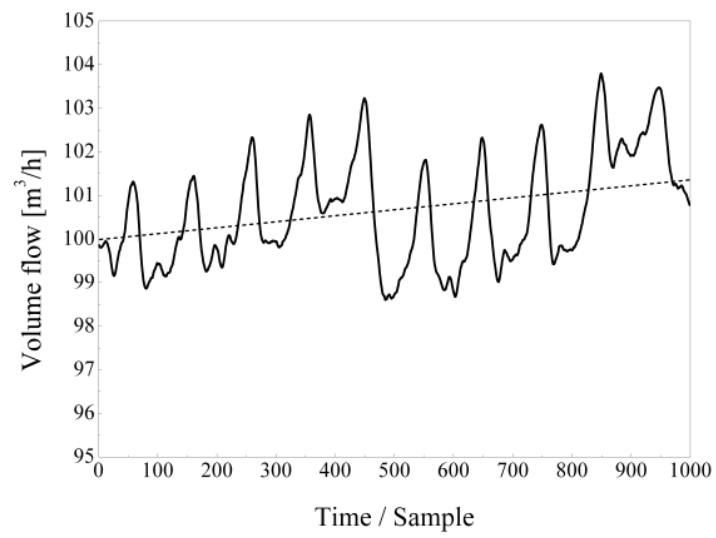

Figure 8. Processed data III by 7-point quadratic polynomial.

In all, it appears from comparing these results that there is a reasonable agreement between the original and processed signal data. However, the standard deviation of the processed data is much lower than the original one. It shows that this smoothing fitter method is efficient.

\section{Conclusions}

In order to obtain improved velocity data from the vortex flow meter, the signal filter method is applied here based on the simple Savitzky-Golay smoothing filter algorithm. The data is computed through the algorithm numerical program. Comparing the processed data between 5-point and 7-point quadratic polynomial filters, it shows that the similarly total average and standard deviation are obtained. The processed data is improved and could be accepted for the analysis. Moreover the filter is acceptable for the linear increase trend data and could prevent the lag error of the flow meter signals. However, due to the property of the vortex flow, the range of the suitable measurement in one meter is strictly limited. The filter for larger range of velocity fluid is under investigation.

\section{Acknowledgements}

The research work was supported by Science and Technology Research Project of Department of Education in Jiangxi Province under Grant No. GJJ151259.

\section{References}

1. A. Venugopal, A. Agrawal and S. V. Prabhu, SENSOR ACTUAT A-PHYS 170, 8-23 (2011).

2. J. E. Amadi-Echendu and H. Zhu, IEEE T INSTRUM MEAS 41, 1001-1006(1992).

3. H. Sato and K. Watanabe, IEEE T INSTRUM MEAS 49, 2002-2005(2000).

4. T. Ghaoud and D. W. Clarke, FLOW MEAS INSTRUM 13, 103-117 (2002).

5. A. G. Rossberg, P. Riegler, F. Buhl, J. Herwig and J. Timmer, FLOW MEAS INSTRUM 15, 2935 (2004).

6. J. J. Miau, C. F. Yeh, C. C. Hu and J. H. Chou, FLOW MEAS INSTRUM 16, 397-404 (2005).

7. D. Zheng, T. Zhang, J. Xing and J. Mei, MEAS SCI TECHNOL 18, 2769 (2007).

8. X. Lin, Y. Wang and Z. Pan, ADV INTE SOFT C 105, 77-82(2011).

9. A. Savitzky and M. J. E. Golay, ANAL CHEM 36, 1627-1639 (1964).

10. J. D. Hunter, F. Pérez and B. E. Granger, COMPUT SCI ENG 13, 13-21(2010). 